Invest. Pens. Crit.(ISSN 1812-3864; eISSN 2644-4119)

Vol. 10, No. 1, Enero - Abril 2022. pp. 51 -58

DOI: https://doi.org/10.37387/ipc.v10i1.275

Comunicación Corta

\title{
Un acercamiento a la noción de alfabetización en energía
}

\author{
An approach to the notion of energy literacy
}

\author{
Manuel Dixon-Pineda ${ }^{1,2^{*}}$ \\ ${ }^{1}$ Doctorando en Educación. Facultad de Educación. Universidad del Istmo. Panamá \\ ${ }^{2}$ Departamento de Química. Facultad de Ciencias Naturales y Exactas. Universidad Autónoma de \\ Chiriquí, Panamá \\ https://orcid.org/0000-0003-0978-3462
}

* Autor por correspondencia: manuel.dixon@unachi.ac.pa

Recibido: 08 de septiembre de 2021

Aceptado: 18 de noviembre de 2021

\section{Resumen}

Actualmente estamos en un mundo cambiante debido al cambio climático, lo que obliga a que el uso de los recursos, incluyendo la energía, fundamental para el desarrollo humano deba ser sustentable en el tiempo. Por ende, es importante comprender cómo es el consumo de energía de las personas. En el presente artículo se analiza un concepto de la educación ambiental que puede ayudar a comprender la compleja relación entre el comportamiento humano y el uso de la energía: la alfabetización en energía. Aquí se presenta un primer acercamiento a este concepto que evalúa de dónde surge y cómo nos puede servir en el futuro, utilizando como ejemplo el caso del consumo energético en Panamá.

Palabras clave: alfabetización en energía, educación ambiental, consumo de energía, alfabetización científica, alfabetización tecnológica.

\begin{abstract}
We are currently in a changing world due to climate change, which requires that the use of resources, including energy, essential for human development, must be sustainable over time. Therefore, it is essential to understand people's energy consumption. This article analyzes a concept of environmental education that can help us understand the complex relationship between human behavior and the use of energy: energy literacy. Here is the first approach to this concept that evaluates where it arises and how it could serve us in the future using the case of energy consumption in Panama.
\end{abstract}

Keywords: energy literacy, environmental education, energy consumption, scientific literacy, technology literacy. 


\section{Introducción}

Los recursos energéticos de un país son propios de sus circunstancias ambientales y socioeconómicas, lo que hace que toda propuesta para su acceso y conservación sea particular, acorde con dichas circunstancias. Desde el punto de vista educativo, existe evidencia de que la educación en energía da un aporte sustancial a todo esfuerzo para promover un consumo responsable de la energía del cual se dispone, independientemente de las condiciones energéticas particulares (Dias et al., 2004), Gill y Lang (2018) estudiaron de forma empírica el efecto que tiene un programa escolar sobre energía en el consumo familiar de electricidad de estudiantes que asistieron al programa y reportaron una reducción aproximadamente del $8 \%$ en el consumo diario, sin embargo también mencionan que esta reducción no era de amplia duración. Sobre el mismo tema, Zografakis et al. (2008) menciona que la educación es una variable importante para lograr cambios de costumbres en el uso de la energía.

Es decir, la educación viene a constituirse en un factor determinante que redunda en beneficio de las condiciones energéticas de una sociedad, siempre y cuando esta responda, igualmente, a las circunstancias particulares en que se genera y accede a la energía, tomando en cuenta las condiciones socioeducativas de determinada sociedad.

Por otro lado, De Alba y González Gaudiano (1997) indican que cada territorio, país o región geográfica requiere de una propuesta particular centrada en sus condiciones particulares, más aún cuando se trata de educar para el enfrentamiento creativo e innovador de los problemas ambientales. Abogan que al momento de desarrollar una propuesta educativa debe estar fundamentada en el contexto en el que se quiere aplicar.

En Panamá actualmente no se cuenta con información acerca de consumo de energía de la población. Por tal motivo, se hace de importancia estudiar este consumo desde una óptica educativa para generar información que sirva de apoyo a las iniciativas educativas propuestas del Estado, establecidas en el Plan Estratégico Energético Nacional.

La manera por la cual se puede estudiar este contexto es con la ayuda del concepto al que se llamará alfabetización en energía, el cual se va a describir en los siguientes apartados.

\section{La alfabetización científica y tecnológica}

La sociedad del siglo XXI se encuentra cada vez más dependiente de sus avances tecnológicos y científicos, lo que causa en el mundo actual una demanda de conocimiento científico y tecnológico para la toma de decisiones comunes mayor. (Cajas, 2001)

Cajas, (2001) indica que paradójicamente a pesar de que nuestras sociedades se sostienen sobre las bases de avances científicos y tecnológicos, ni las sociedades autollamadas desarrolladas tienen clara la naturaleza de la ciencia y muchos menos de la tecnología. Este hecho, hace que la falta del conocimiento científico y tecnológico útil dificulte que los ciudadanos comprendan y transformen la realidad que los rodea.

De acuerdo con Cajas, (2001) la alfabetización científica puede ser vista como una transposición didáctica entendida como movimiento de los saberes científicos a saberes escolares. De acuerdo con el autor antes mencionado la dificultad relacionada a la trasposición didáctica se encuentra en que el mecanismo por el cual se realiza este movimiento actualmente no se entiende con claridad entendido debido a la complejidad; sin embargo, para los fines de la alfabetización científica y tecnológica de la población en general considera que lo importante no es la transposición didáctica de todo el conocimiento disponible sino de aquellos conocimientos que puedan ser relevantes para todos.

De acuerdo con la propuesta de Cajas lo importante del proceso de transposición didáctica es que la información que llega a los estudiantes escolares le sea útil para la toma de decisiones en la vida diaria. Esto significa que debe ser de interés estudiar la alfabetización científica y tecnológica de las personas para determinar que saberes científicos son objeto de la transposición didáctica los estudiantes.

Al estudiar el proceso de la alfabetización científica se puede entender de mejor manera cómo es la transposición didáctica de forma que se puede optimar la efectividad de propuestas educativas dirigidas a mejorar la capacidades científicas-tecnológicas-sociales de las personas para asegurar un desarrollo sostenible en el tiempo.

Para Cañal, (2004):

"Es evidente que la alfabetización científica no es un proceso espontáneo que pueda producirse en alguien por simple inmersión en la cultura común de nuestra sociedad. La perspectiva de la ciencia 
no es más que una de las diversas componentes del saber disponible, y se trata de un sector cultural extenso, complejo y recientemente desarrollado, en relación a otros, y por ello aún escasamente asimilado e integrado en el conocimiento compartido de la población. El proceso de enculturación científica depende en gran manera, por todo lo anterior, de la actuación de las instituciones educativas y de los medios de comunicación social." (p.246)

Para terminar la presentación del concepto de alfabetización científica se presenta a Marco-Stiefel, (2004) que indica lo siguiente:

"La alfabetización refiere, en cualquier diccionario, a las capacidades más básicas que permiten acceder a la realidad, ordinariamente, la lectura y la escritura, o lo que es lo mismo: la adquisición de rudimentos básicos para poderse entender y comunicar. Traspasando esta acepción al campo científico podemos decir que la alfabetización científica comporta la adquisición de rudimentos básicos en orden a la comprensión de la ciencia y de la tecnología de modo que se puedan utilizar esos conocimientos en la comunicación y la argumentación con base científica y, llegado el caso, en el pronunciamiento sobre los temas científicos y sus derivaciones en lo que es propio de la acción ciudadana”. (p. 274)

A partir de los anteriores conceptos se analizará el tema de la alfabetización en energía como un objeto de la alfabetización científica y tecnológica.

\section{Alfabetización científica + Alfabetización tecnológica = Alfabetización en Energía}

En el apartado anterior se presentó que la mecánica de estudio del movimiento de saberes científicos a sabres escolares es por medio de la alfabetización científica y tecnológica. Si nuestro interés es estudiar el movimiento de saberes científicos y tecnológicos en un tema como lo es la energía, y considerando que la energía y su uso, es una mezcla de saberes científicos y tecnológicos, por lo tanto, al estudiar el movimiento de saberes relacionados con la energía y su uso podríamos hablar de Alfabetización en Energía en nuestro idioma español. Por ejemplo, en el idioma inglés se utiliza el término "Energy Literacy" que de acuerdo con el Departamento de Energía de EE. UU., (2014) hace referencia a la capacidad que tienen las personas para entender cómo afecta la vida el uso de la energía y también cómo utilizar estos conocimientos para resolver problemas asociados a ella. Debe notarse que en el idioma inglés ya se cuenta con un término asociado para el estudio del movimiento de los saberes científicos a escolares en cuanto a términos de energía se refiere.

Así mismo, El Departamento de Energía de EE. UU., (2014) menciona que una persona versada en temas de energía tiene las siguientes características:

- Puede rastrear flujos de energía y pensar en términos de sistemas de energía.

- Sabe cuánta energía se usa, con qué propósito y de dónde proviene la energía.

- Puede evaluar la credibilidad de la información sobre la energía.

- Puede comunicarse sobre la energía y el uso de energía de manera significativa.

- Es capaz de tomar decisiones informadas sobre el uso de energía basadas en la comprensión de los impactos y las consecuencias.

La alfabetización en energía busca estudiar la relación que existe entre el uso racional de la energía y la educación. El campo de estudio de la alfabetización en energía cuenta con diferentes puntos de vista los cuales no son excluyentes, sino que cada uno busca aportar al entendimiento del proceso de cómo se estudian las competencias de las personas sobre el tema del uso adecuado de la energía y también se busca estudiar cómo se pueden optimizar estas competencias en la población general, de manera que nuestras sociedades sean sostenibles en el tiempo.

Martins et al., (2019) en su artículo de revisión presenta las principales corrientes de pensamiento en el estudio de la alfabetización en energía, entre cuales se encuentran:

1. Relación cognitiva-actitud-conductual del uso de la energía.

2. Enfoque de tipo económico sobre el costo beneficio a largo plazo de invertir en equipos eficientes.

3. Conciencia propia del consumo de energía y la conciencia de adoptar conductas ahorrativas de energía.

4. Necesidad del conocimiento y de las destrezas en el procesamiento de la información para hacer cálculos financieros relacionados al uso de energía.

De las corrientes antes mencionadas una que presenta particular interés para nosotros es la corriente de pensamiento la cual fue propuesta por J. E. DeWaters y Powers, (2008), en la que la definición de la alfabetización en energía tiene una óptica educativa que se fundamenta en los componentes de la educación 
ambiental y además estos investigadores desarrollaron un instrumento que permite estudiar la alfabetización en energía de jóvenes adolescentes. A continuación, se va a realizar una presentación del instrumento propuesto por DeWaters y colaboradores.

\section{DeWaters, colabores y el instrumento para estudiar la alfabetización en energía}

Desde el año 2008 DeWaters y colaboradores realizaron estudios para desarrollar un instrumento que permitiera evaluar la alfabetización en energía de jóvenes adolescentes. Este instrumento de evaluación está compuesto por tres niveles que se encuentran fundamentados en los preceptos de la Educación Ambiental entre los que se pueden mencionar:

- Nivel Conocimiento: que es la comprensión de conceptos científicos básicos, reglas y teorías, transferencia de energía y procesos de transformación, la influencia de los flujos de energía y el papel que desempeña la energía en los ecosistemas.

- Nivel Actitud: que evalúa la comprensión del suministro de energía común y las situaciones de cierre, los procesos de producción y uso de energía y los consiguientes impactos ambientales, la influencia de los problemas energéticos en la vida humana y las convicciones e ideologías de cada persona, basadas en el conocimiento energético, que son inherentes al proceso de toma de decisiones.

- Nivel Comportamiento: que evalúa la conciencia personal del impacto de las acciones cotidianas, la producción y el uso de la energía, la responsabilidad de cada uno como ciudadano del mundo y el compromiso con acciones efectivas y verdaderamente comprometidas en el ahorro de energía.

Los trabajos en donde se presenta el desarrollo de instrumento se encuentran publicados en DeWaters et al., (2013); DeWaters y Powers, (2011); DeWaters y Powers, (2008, 2013).

A continuación, se muestra un esquema del sustento conceptual del instrumento desarrollado por estos autores:

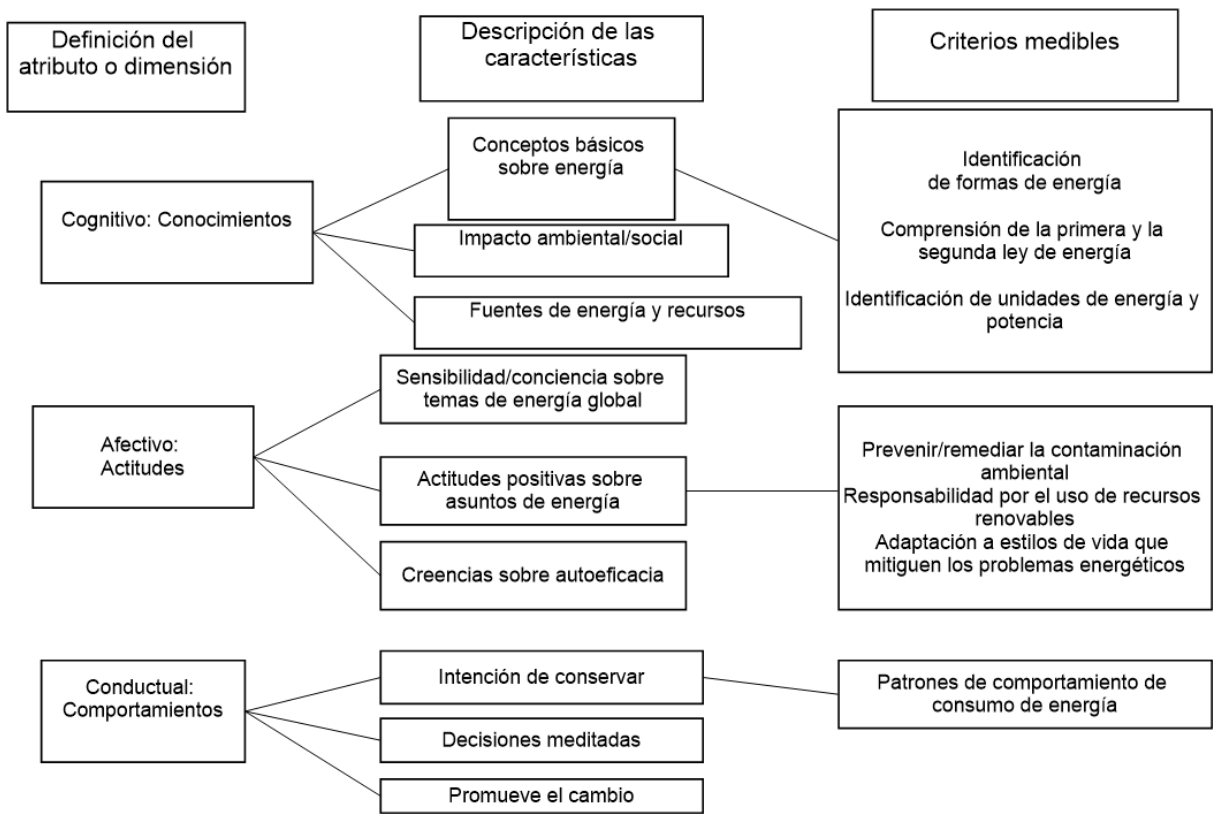

Figura 1. Criterios para la Alfabetización en energía: atributos de ejemplo, características y puntos de referencia medibles. Fuente: Traducción libre de DeWaters \& Powers, (2008)

Los niveles antes descritos son las variables que se utilizan para la operacionalización del proceso de evaluación de la alfabetización en energía las cuales se describen a continuación:

- Cognitiva: conocimiento, comprensión, habilidades cognitivas y de resolución de problemas.

- Afectiva: conciencia, actitudes, valores, locus de control, sentido de responsabilidad personal.

- Comportamiento: participación, habilidades de acción.

El producto de los estudios de DeWaters et. al, (2013), J. E. DeWaters \& Powers, (2013, 2011b) es un 
cuestionario de ochentaicinco (85) preguntas que se encuentra divido de la siguiente manera: cincuenta (50) preguntas son de nivel cognitivo las cuales se evalúan escogiendo la opción correcta y treintaicinco (35) preguntas de entre el nivel afectivo y conductual con escala de evaluación tipo Likert de 5 puntos.

Debe notarse que el instrumento de medición antes descrito permite estudiar las tres áreas que componen la alfabetización energética lo que facilita su tratamiento matemático y estadístico, al mismo tiempo nos permite poner a prueba posibles hipótesis que se tengan sobre como incide una variable sobre otra o si existe correlación entre variables de interés.

\section{Estudios sobre la alfabetización en energía.}

En este apartado se va a realizar una descripción de estudios en el mundo en los que se ha utilizado el instrumento desarrollado por DeWaters y colaboradores. En cuanto al instrumento final de trabajo siempre que se menciona que se utilizó como base de construcción el instrumento desarrollado por Dewaters y colaboradores sin embargo algunas de las preguntas propuestas fueron modificadas para ajustar el instrumento a la realidad regional en donde se realizaba el estudio.

Como primer ejemplo se mencionará el trabajo de Oo et al., (2017) con 225 estudiantes de secundaria en Nigeria concluyen que si bien en términos generales los estudiantes encuestados demostraron conocimiento básico sobre temas generales de energía, el conocimiento de los estudiantes sobre la realidad energética y uso de la energía eléctrica en su país es bajo.

El 88,5\% de los estudiantes encuestados apoya la introducción de la educación energética en el currículo del Colegio Secundario en Nigeria. En cuanto a la actitud energética más del 80\% de los encuestados apoyan la eficiencia y la conservación energéticas. Una recomendación interesante realizada al final del trabajo consiste en que se debe desarrollar una política energética y que esta política debe hacer que la educación en energía sea parte fundamental del currículo educativo en Nigeria con una mayor atención al uso de fuentes renovables de energía.

Aguirre-Bielschowsky et al., (2017) en Nueva Zelanda exploraron el proceso involucrado en el desarrollo de la alfabetización energética en niños en dos contextos la escuela y el hogar. En este estudio también se buscaba estudiar el potencial de los niños de actuar como agentes de conservación de energía específicamente influenciando a su familia para ahorrar electricidad.

Entre algunos de los hallazgos de este trabajo mencionan que en el caso de los niños entrevistados ninguno había desarrollado completamente las tres dimensiones de la alfabetización energética propuesta por J. DeWaters \& Powers,(2008). Los autores del estudio al argumentan que la falta de conocimiento que presentan los niños es un reflejo de la población general probablemente sea el resultado de su dependencia de fuentes de aprendizaje informales tales como: sus padres, que en mucha de las ocaciones no se preocupan por la conservación de energía o simplemente no son capaces de explicar problemas complejos relacionados al uso de la energía, y otro son los medios de comunicación que suelen ofrecer información irregular y fuera de contexto. En conclusión este tipo de estudio deja en evidencia la importancia de incorporar la educacion en energía en las escuelas porque una forma estructurada de aprendizaje puede ayudar a mejorar de forma efectiva los conocimientos, actitudes y conductas de consumo de energía de los niños.

En Taiwan Lee et al., (2015) se utlizó de base el cuestionario desarrollado por DeWaters y colaboradores ajustado al contexto del país incluyendo traducción.

Entre los resultados encotrados de este estudio se menciona que el nivel de alfabetización en energía de los jóvenes taiwaneses es alto. Esto se contrapone con el hallazgo hecho por J. E. DeWaters y Powers, (2011b) en EE.UU. en donde el nivel de alfabetización en energía es bajo. Lee et al. atribuyen la diferencia posiblemente a que Taiwan es un país con recursos insuficientes y no es un país con independencia energética, lo que hace que sus habitantes estén en mayor sintonía con la conservación y reducción de la huella de carbono de manera que sea ambietalmente sustentables.

\section{La alfabetización en energía en Panamá y por qué estudiarla.}

En Panamá la entidad encargada de los temas relacionados con la energía es la Secretaría Nacional de Energía (SNE), que desarrolló el Plan Estratégico Nacional de energía (PEN 2015-2050) publicado en la Gaceta Oficial Digital 28003-A en 2016. En este documento se establecen los planes y metas a cumplir para el país para el año 2050. También en el documento se menciona que la SNE promoverá el uso racional de la energía por 
medio de la educación a la población. En el plan estratégico energético de la SNE en la sección de energía y educación menciona lo siguiente:

"La implementación de Plan Energético Nacional tiene también una dimensión didáctica y educativa, que guarda relación con la formación de una nueva cultura del consumo racional y ético de los recursos naturales (no sólo la energía) y con el cambio de actitud de los ciudadanos ante el medio ambiente. La sociedad de la información en la que vivimos ofrece los medios técnicos para lograr el cambio cultural que debe complementar las leyes y las propuestas de política energética a largo plazo. Al igual que otros temas de afectación transversal, los aspectos educativos tocan todas las actividades humanas. En el caso del sector energético se deben coordinar con las autoridades y organismos competentes, en primer lugar, con el Ministerio de Educación y autoridades académicas de los centros de educación superior. También se coordinará con programas similares que adelantan otras instituciones públicas como el MIAMBIENTE y otros organismos no gubernamentales. La SNE considera que la educación es un proceso de dos vías, en el sentido que la información fluirá en dos sentidos: desde la SNE a la sociedad a través de información actualizada y distribuida por los medios de comunicación más efectivos; pero también servirá para abrir un canal mediante el cual la sociedad en haga llegar información a la SNE sobre las necesidades de información y de la forma en que esta percibe el problema de la energía”. (pág. 305)

Pero el tema no solo ha sido abordado desde el punto de vista conceptual y educativo sino político también como es el caso de Panamá país en el que, a partir del año 2012, se cuenta con la ley 69 del 12 de octubre llamada UREE (Ley de Uso Racional y Eficiente de Energía) que en su primer artículo menciona que esta ley establece los lineamientos generales de la política nacional para el uso racional y eficiente de la energía en el territorio nacional.

En vista entonces de que, para la SNE la educación puede ser un vehículo para producir cambios en los hábitos de consumo y uso de la energía, se hace de importancia estudiar el consumo de energía de la población desde una óptica educativa para generar información que sirva de apoyo a las iniciativas educativas propuestas del Estado, establecidas en el Plan Estratégico Energético Nacional.

Para el estudio de las competencias de nuestros habitantes sobre temas relacionados con energía se requerirá recurrir a los aportes teóricos y prácticos propios del enfoque de la alfabetización en energía además de recurrir a una especie de diagnóstico que permita reconocer las capacidades de amplio espectro, o competencias de la población.

Examinar las capacidades de nuestros habitantes sobre temas relacionados con la energía (alfabetización en energía) es de vital importancia porque los resultados empíricos obtenidos servirán de insumo para la creación de principios pedagógicos para el desarrollo e implementación de planes educativos con la finalidad de concientizar a los panameños.

Este tipo de investigación brindará los fundamentos pedagógicos para la elaboración de propuestas educativas contextualizadas sobre Educación Ambiental en el campo específico de la alfabetización en energía.

Toda propuesta de educación para ser efectiva debe ser desarrollada desde la realidad local del problema que se desea atender. En nuestro caso si el problema que se desea atender es el uso racional de la energía lo primero que se debe hacer es describir las concepciones, prácticas y compromiso afectivo de los sujetos de la investigación, es decir su estado de alfabetización en energía.

En Panamá actualmente no se cuenta con información acerca de consumo de energía de la población. Por tal motivo se hace de importancia estudiar este consumo desde una óptica educativa para generar información que sirva de apoyo a las iniciativas educativas propuestas del Estado, establecidas en el Plan Estratégico Energético Nacional.

En una primera fase del estudio de la alfabetización en energía en el país se debe concretar el marco conceptual que articule la situación de Panamá.

El siguiente paso propuesto en el estudio consistirá en la adaptación del instrumento propuesto por J. DeWaters y Powers, (2013) al contexto nacional. Por lo tanto, será necesaria su traducción del inglés al español, contextualización y validación. En la adaptación del instrumento guía a nuestra realidad se debe verificar que las preguntas a utilizar respondan a:

1. la realidad energética de nuestro país (fuentes de generación de energía que en el caso de Panamá la mayoría son renovables).

2. contraste con las leyes vigentes sobre uso racional de energía (Ley UREE: Ley Uso Racional y Eficiente de Energía).

3. propuestas sobre uso de energía y educación establecidas en el PEN por la Secretaría de Energía de 
Panamá.

4. lineamientos sobre educación ambiental establecidos en el MEDUCA y MiAmbiente.

Además, se debe estudiar la alfabetización en energía en las diferentes regiones del país para que al momento que se planteen propuestas educativas éstas, se encuentren contextualizadas a las necesidades del medio en el que se plantean utilizar.

\section{Conclusiones}

Para Panamá el estudio de la alfabetización en energía puede ser la herramienta que ayude a comprender como son las competencias de nuestra población sobre temas relacionados de energía (uso racional y eficiente).

Con la información recopilada sobre la alfabetización en energía se puede generar los fundamentos básicos requeridos para generar $\mathrm{u}$ optimizar propuestas educativas contextualizadas que apoyen las iniciativas propuestas por la Secretaría de Energía de Panamá.

\section{Agradecimientos}

Agradezco a la Dra. Alejandrina Mata-Segreda y la Dra. Nadia de León por su apoyo en el desarrollo de este manuscrito.

\section{Referencias}

Aguirre-Bielschowsky, I., Lawson, R., Stephenson, J., \& Todd, S. (2017). Energy literacy and agency of New Zealand children. Environmental Education Research, 23(6), 832-854. https://doi.org/10.1080/13504622.2015.1054267

Cajas, F. (2001). Alfabetización científica y tecnológica. Ensenanza de las Ciencias, 19(2), 243-254. http://ddd.uab.cat/record/1528

Cañal, P. (2004). La alfabetización científica: ¿necesidad o utopía? Scientific literacy : A need or and ideal? Cultura y Educación, 16(3), 245-257.

De Alba, A,; González Gaudiano, E. (1997) Evaluación de programas de educación ambiental. México: UNAM.

Departamento de Energía de EE. UU. (2014). Conocimiento de Energía-Principios Esenciales y Conceptos Fundamentales para la Educación de Energía (pp. 1-20). https://www.energy.gov/sites/default/files/2015/04/f21/ConocimientoEnergia_high_res.pdf

DeWaters, J. E., \& Powers, S. (2008). Energy literacy among middle and high school youth. Proceedings Frontiers in Education Conference, FIE, November. https://doi.org/10.1109/FIE.2008.4720280

DeWaters, J. E., \& Powers, S. (2013). Establishing measurement criteria for an energy literacy questionnaire. Journal of Environmental Education, 44(1), 38-55. https://doi.org/10.1080/00958964.2012.711378

DeWaters, J. E., \& Powers, S. E. (2011a). Energy literacy of secondary students in New York State (USA): A measure of knowledge, affect, and behavior. Energy Policy, 39(3), 1699-1710. https://doi.org/10.1016/j.enpol.2010.12.049

DeWaters, J. E., \& Powers, S. E. (2011b). Energy literacy of secondary students in New York State (USA): A measure of knowledge, affect, and behavior. Energy Policy, 39(3), 1699-1710. https://doi.org/10.1016/j.enpol.2010.12.049

DeWaters, J., \& Powers, S. (2008). Energy literacy among middle and high school youth. Proceedings Frontiers in Education Conference, FIE, November. https://doi.org/10.1109/FIE.2008.4720280

DeWaters, J., \& Powers, S. (2013). Establishing measurement criteria for an energy literacy questionnaire. Journal of Environmental Education, 44(1), 38-55. https://doi.org/10.1080/00958964.2012.711378

DeWaters, J., Qaqish, B., Graham, M., \& Powers, S. (2013). Designing an energy literacy questionnaire for middle and high school youth. Journal of Environmental Education, 44(1), 56-78. https://doi.org/10.1080/00958964.2012.682615

Dias, R. A., Mattos, C. R., \& Balestieri, J. A. P. (2004). Energy education: Breaking up the rational energy use barriers. Energy Policy, 32(11), 1339-1347. https://doi.org/10.1016/S0301-4215(03)00100-9

Gill, C., \& Lang, C. (2018). Learn to conserve: The e ff ects of in-school energy education on at-home electricity consumption. 118(March), 88-96. https://doi.org/10.1016/j.enpol.2018.03.058 
Lee, L. S., Lee, Y. F., Altschuld, J. W., \& Pan, Y. J. (2015). Energy literacy: Evaluating knowledge, affect, and behavior of students in Taiwan. Energy Policy, 76, 98-106. https://doi.org/10.1016/j.enpol.2014.11.012

Marco-Stiefel, B. (2004). Alfabetización científica: un puente entre la ciencia escolar y las fronteras científicas. Cultura y Educación, 16(3), 273-287. https://doi.org/10.1174/1135640042360906

Martins, A., Madaleno, M., \& Ferreira, M. (2019). Energy literacy: What is out there to know? Energy Reports, xxxx, 6-11. https://doi.org/10.1016/j.egyr.2019.09.007

Oo, B., Abbasoglu, S., Dagbasi, M., \& Garba, M. (2017). Evaluation of Energy Literacy among Nigerian Senior Secondary Students. Research \& Reviews: Journal of Educational Studies Evaluation, 3(1), 1118.

Zografakis, N., Menegaki, A. N., \& Tsagarakis, K. P. (2008). Effective education for energy efficiency. Energy Policy, 36, 3226-3232. https://doi.org/10.1016/j.enpol.2008.04.021 